\title{
Study on interaction between salicylaldehyde I-serine schiff base and human serum albumin by fluorescence spectroscopy
}

\author{
Yanqiu Yang, Yanju Liu, Jundi Zhang and Huaixia Yang ${ }^{a}$ \\ School of Pharmacy, Henan University of Chinese Medicine, Zhengzhou 450046, China
}

\begin{abstract}
The interaction of salicylaldehyde L-serine Schiff base (L) with human serum albumin (HSA) was examined by fluorescence emission spectra at the excitation wavelength $290 \mathrm{~nm}$. Through fluorescence quenching experiments, it was confirmed that the combination of $\mathrm{L}$ with HSA was static quenching process. Thermodynamic parameters, such as $\Delta \mathrm{G}, \Delta \mathrm{H}$ and $\Delta \mathrm{S}$, were calculated at different temperatures, showing that van der Waals force or hydrogen bond interaction were mostly responsible for the binding of L to HSA. The experiments results showed that the microenvironment and the conformation of HSA changed during the binding reaction.
\end{abstract}

\section{Introduction}

Amino acid Schiff base attracted attention of researchers, for their interesting structure and unique biological action. Serum albumin can be effectively combined with many type endogenous substances in the human body and exogenous drugs, which play an important role in storage and transportation [1]. Therefore, the study of the interaction between proteins and drug molecules are a meaningful topic in the field of life and chemical sciences, which help to study the strength of the combination and the bonding mechanism between proteins and small molecules.

In the present work, the interaction between L and HSA was investigated at two temperatures under physiological conditions, utilizing the fluorescence method. We probed into the fluorescence quenching mechanism of HSA by $\mathrm{L}$ and calculated the binding constant $K_{\mathrm{b}}$ and the number of binding sites $\mathrm{n}$ for L-HSA complex.

\footnotetext{
${ }^{a}$ Corresponding author: yanghuaixia886@163.com

This study was supported by HenanProvince basic and frontier technology researchprojects (152300410214), Program for the key scientific research project of colleges and universitiesin Henan Province (16A350002), and Science \& Technology Innovation Talents in Henan College of Chinese Medicine (2014XCXRC04).
} 


\section{Experimental}

\subsection{Apparatus and reagent}

\subsubsection{Apparatus}

970 CRT fluorometry (Shanghai Scientific Instrument Co., Ltd. China), MP521 Lab pH/Conductivity Meter (Shanghai San-Xin Instrumentation, Inc. China).

\subsubsection{Reagents}

Salicylic aldehyde (Sinopharm Group Co., Ltd. China), L-serine (Biochemical reagents, Sinopharm Group Co., Ltd. China), HSA (Beijing Olympic nemesis Biological Technology Co. Ltd. China, relative molecular mass 66000$)$, was dissolved daily in $0.1 \mathrm{~mol} \cdot \mathrm{L}^{-1}$ Tris- $\mathrm{HCl}$ buffer solution $(\mathrm{PH}=7.4)$ to prepare a solution $\left(1 \times 10^{-5} \mathrm{~mol} \cdot \mathrm{L}^{-1}\right)$, salicylaldehyde L-serine Schiff base to prepare a solution $\left(1.0 \times 10^{-3} \mathrm{~mol} \cdot \mathrm{L}^{-1}\right)$, All chemicals were analytical-reagent grade. All solutions were prepared with double-distilled water.

\subsection{Methods}

$\mathrm{L}$ is synthesized on the basis of reference [2]. The synthesis route shown as Figure 1.<smiles>NC(CO)C(=O)c1cccc(O)c1C=NC(C=O)CO</smiles>

Figure 1. The synthesis route of L.

An appropriate concentration of HSA was titrated by successive additions of a $1.0 \times 10^{-3} \mathrm{~mol} \cdot \mathrm{L}^{-1}$ doubly distilled water stock solution of L. Titrations were done manually by using trace syringes. The fluorescence and Synchronous fluorescence spectra was measured on 970 CRT fluorometry, using 1.0 $\mathrm{cm}$ quartz cells. The excitation wavelength was $290 \mathrm{~nm}$. the $\Delta \lambda$ is set at $60 \mathrm{~nm}$ or $15 \mathrm{~nm}$. Experiments were measured under two temperatures $(298 \mathrm{~K}, 308 \mathrm{~K})$.

\section{Results and discussion}

\subsection{Fluorescence quenching of HSA}

The fluorescence of HSA originates from the amino acid residues of tryptophan (Trp) and tryosin (Tyr), which are sensitive to the environment around them [3]. It emits a strong fluorescence peaked at $352 \mathrm{~nm}$ with excitation wavelength of $290 \mathrm{~nm}$. Figure 2 shows the fluorescence quenching spectra of solutions containing HSA fixed concentration and different concentrations of L at $298 \mathrm{~K}$ and $308 \mathrm{~K}$, it can be observed that the fluorescence intensity of HSA decreases regularly with increasing of L, but there is no significant emission wavelength shift. These results suggest that L can interact with HSA and quench its intrinsic fluorescence [4]. 

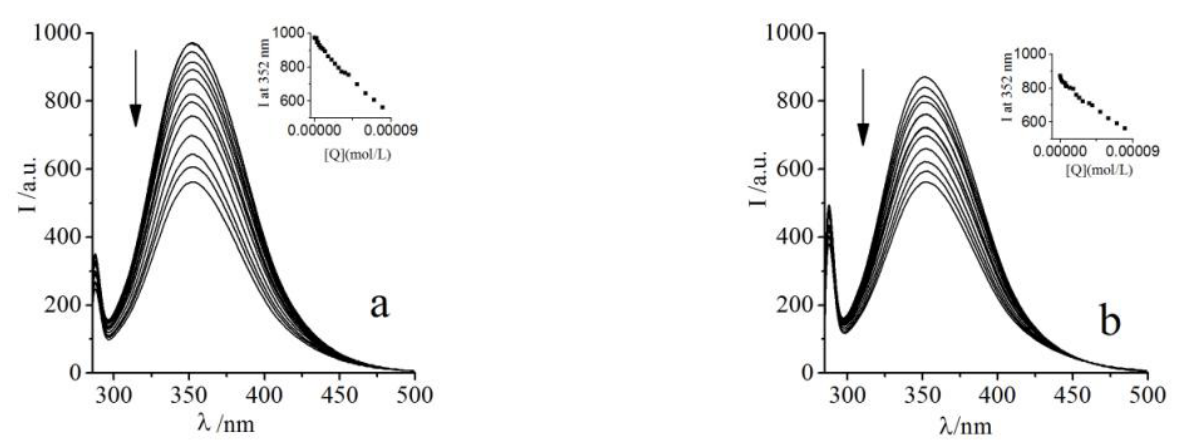

Figure 2. Changes in the emission spectra $(\lambda e x=290 \mathrm{~nm})$ of the HSA $\left(1 \times 10^{-5} \mathrm{~mol} / \mathrm{L}\right)$ upon increasing concentrations of L (a) $298 \mathrm{~K}$ and (b) $308 \mathrm{~K}$.

\subsection{Determination of quenching mechanism}

There are two quenching types in characterizing the mechanism of the interaction between quenchers and macromolecules: static and dynamic quenching. Static quenching refers to the formation of a nonfluorescence fluorophore-quencher complex. Dynamic (collisional) quenching refers to the quencher diffusing towards the fluorophore during the lifetime of the excited state and, upon contact, the fluorophore returns to the ground state without emission of a photon. Dynamic quenching mechanism was probed using the Stern-Volmer equation [5].

$$
F_{0} / F=1+K_{\mathrm{q}} \tau_{0}[\mathrm{Q}]=1+K_{\mathrm{sv}}[\mathrm{Q}]
$$

where $F_{0}$ and $F$ represent the fluorescence intensities of HSA in the absence and presence of the external quencher, $K_{\mathrm{q}}$ is the bimolecular quenching rate constant, $K_{\mathrm{sv}}$ is the Stern-Volmer quenching constant, $\tau_{0}$ is the average lifetime of biopolymers without quencher, the fluorescence lifetime of the biopolymers is $10^{-8} \mathrm{~s}[6],[\mathrm{Q}]$ is the concentration of the quencher.

This work studied the fluorescence spectrum of the action between HSA and L, obtained the plot of $F_{0} / F$ against [Q] at different temperature. As it was shown in Figure 3. Equation (1) was applied to determine $K_{\mathrm{sv}}$ by linear regression from a plot of $F_{0} / F$ against [Q], hence, $K_{\mathrm{q}}$ can be caculate by $K_{\mathrm{q}}=$ $K_{\text {sv }} / \tau_{0}$. As shown in Table 1 .
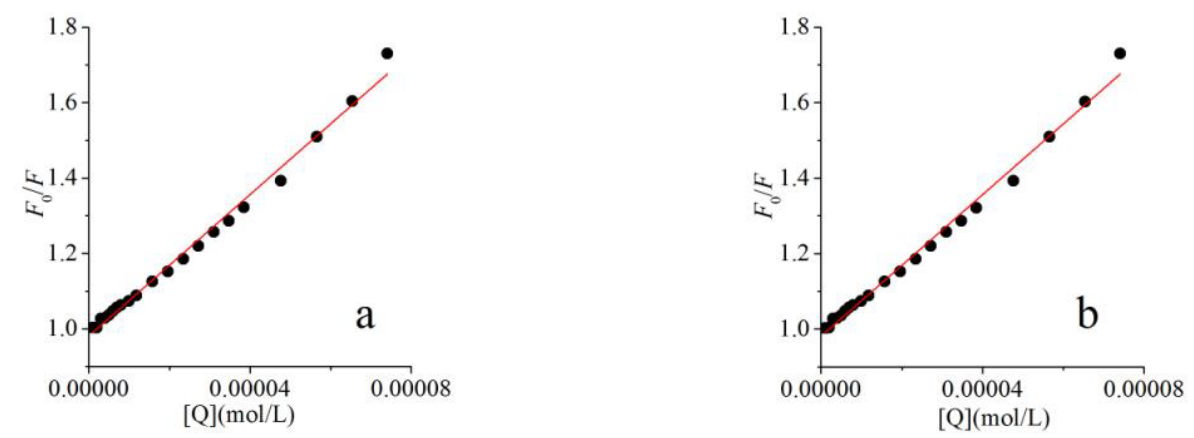

Figure 3. Stern-Volmer plot for the quenching of HSA by L at different temperatures (a)298K and (b)308K. 
Table 1. Constant of the systems of interaction between L and HSA at different temperatures.

\begin{tabular}{ccc}
\hline $\mathrm{T} /(\mathrm{K})$ & $K_{\mathrm{sv}} /\left({\left.\mathrm{L} \cdot \mathrm{mol}^{-1}\right)}^{-1}\right.$ & $K_{\mathrm{q}} /\left(\mathrm{L} \cdot \mathrm{mol}^{-1} \cdot \mathrm{s}^{-1}\right)$ \\
\hline 298 & $9.375 \times 10^{3}$ & $9.375 \times 10^{11}$ \\
308 & $9.397 \times 10^{3}$ & $9.397 \times 10^{11}$ \\
\hline
\end{tabular}

For the L-HSA systems, the values of $K_{\mathrm{sv}}$ and $K_{\mathrm{q}}=K_{\mathrm{sv}} / \tau_{0}$ obtained from the plots at $298 \mathrm{~K}$ and $308 \mathrm{~K}$ are shown in Table 1. According to the literature [7] for dynamic quenching, the maximum scatter collision quenching constant of various quenchers with the biopolymer is $2.0 \times 10^{10} \mathrm{~L} \cdot \mathrm{mol}^{-1} \cdot \mathrm{s}^{-1}$. The values of $K_{\mathrm{q}}$ are larger than $2.0 \times 10^{10} \mathrm{~L} \cdot \mathrm{mol}^{-1} \cdot \mathrm{s}^{-1}$, which suggest that the fluorescence quenching is caused by a specific interaction between HSA and L, and the quenching mechanism mainly arise from complexes formation, while dynamic collision could be negligible in the concentration range studied [8].

\subsection{The binding constant $K_{\mathrm{b}}$ and the number of binding sites $\mathrm{n}$}

For static quenching, the relationship between fluorescence quenching intensity and the concentration of quenchers can be used to obtain the binding constant and the number of binding sites [9].

$$
\operatorname{Lg}\left[\left(F_{0}-F\right) / F\right]=\operatorname{Lg} K_{\mathrm{b}}+\mathrm{nLg}[\mathrm{Q}]
$$

The values of $K_{\mathrm{b}}$ and $\mathrm{n}$ at $298 \mathrm{~K}$ and $308 \mathrm{~K}$ were obtained from the double logarithm regression curve (Figure 4 ). The values of $K_{\mathrm{b}}$ and $\mathrm{n}$ at $298 \mathrm{~K}$ and $308 \mathrm{~K}$ are listed in Table 2.

The $\mathrm{n}$ values are nearly 1 , and thus indicate the existence of a single binding site in HSA for L. The interactions of L with HSA increased when rising the temperature, which suggest that interactions of L with HSA were endothermic.
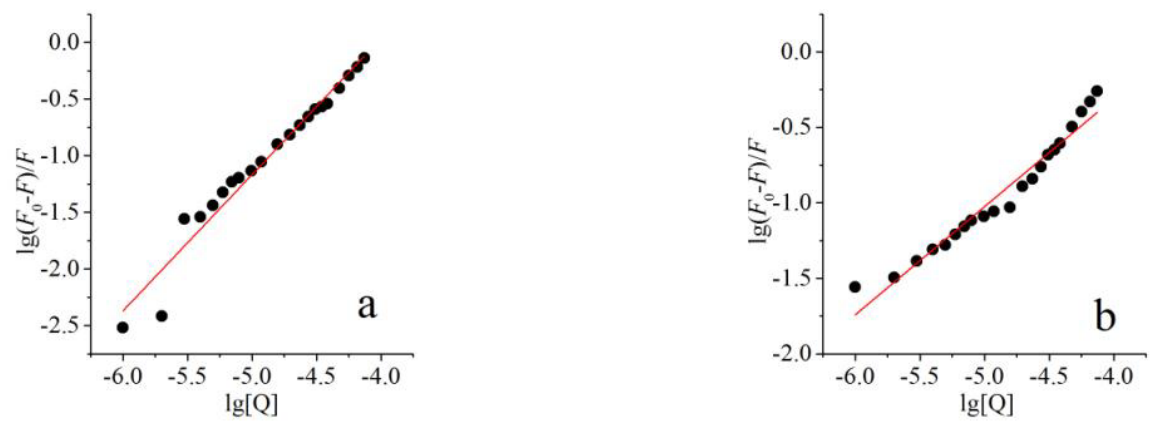

Figure 4. The plots of $\lg \left[\left(F_{0}-F\right) / F\right]$ vs. $\lg [\mathrm{Q}]$ at different temperatures (a) $298 \mathrm{~K}$ and (b) $308 \mathrm{~K}$.

Table 2. The binding constant, $K_{\mathrm{b}}$, and the number of binding sites, $\mathrm{n}$

\begin{tabular}{crccc}
\hline $\mathrm{T} /(\mathrm{K})$ & double logarithm e quation & $\mathrm{R}$ & $K_{\mathrm{b}} /(\mathrm{L} / \mathrm{mol})$ & $\mathrm{n}$ \\
\hline 298 & $\operatorname{Lg}\left[\left(F_{0} / F\right) / F=4.8474+1.202 \mathrm{Lg}[\mathrm{Q}]\right.$ & 0.3211 & $7.0372 \times 10^{4}$ & 1.202 \\
308 & $\operatorname{Lg}\left[\left(F_{0} / F\right) / F=2.5554+0.716 \mathrm{Lg}[\mathrm{Q}]\right.$ & 0.1269 & $0.0359 \times 10^{4}$ & 0.716 \\
\hline
\end{tabular}




\subsection{Binding modes}

The acting forces between a drug and a biomolecule are composed of weak interactions of molecules such as hydrogen bond formation, van der Waals forces, electrostatic forces, and hydrophobic interaction [10]. The thermodynamic parameters could be calculated by eqation (3), (4) and (5),

$$
\begin{gathered}
\operatorname{Ln}\left(\mathrm{K}_{2} / \mathrm{K}_{1}\right)=\Delta \mathrm{rHm}\left(1 / \mathrm{T}_{1}-1 / \mathrm{T}_{2}\right) / \mathrm{R} \\
\Delta \mathrm{rGm}=-\mathrm{RTLnK} \\
\Delta \mathrm{rSm}=(\Delta \mathrm{rHm}-\Delta \mathrm{rGm}) / \mathrm{T}
\end{gathered}
$$

The thermodynamic parameters are presented in Table 3.

Table 3. Thermodynamic parameters for the association of L with HAS.

\begin{tabular}{cccc}
\hline $\mathrm{T} / \mathrm{K}$ & $\Delta \mathrm{rHm} /\left(\mathrm{kJ} \mathrm{mol}^{-1}\right)$ & $\Delta \mathrm{rSm} /\left(\mathrm{J} . \mathrm{K}^{-1}\right)$ & $\Delta \mathrm{rGm} /\left(\mathrm{kJ} . \mathrm{mol}^{-1}\right)$ \\
\hline 298 & & & -27.66 \\
308 & -402.80 & -1258.86 & -15.07 \\
\hline
\end{tabular}

The negative signs for $\Delta \mathrm{G}$ reveal that the binding processes are spontaneous, the formation of drug-HSA coordination compounds are spontaneous. The thermodynamic parameters, enthalpy change $(\Delta \mathrm{H})$ and entropy change $(\Delta \mathrm{S})$ are the main evidence to confirm binding modes. From the thermodynamic standpoint, $\Delta \mathrm{H}>0$ and $\Delta \mathrm{S}>0$ imply a hydrophobic interaction; $\Delta \mathrm{H}<0$ and $\Delta \mathrm{S}<0$ reflect the van der Waals forces or hydrogen bond formation; and $\Delta \mathrm{H} \approx 0$ and $\Delta \mathrm{S}>0$ suggest an electrostatic force [11]. The both negative $\Delta \mathrm{H}$ and $\Delta \mathrm{S}$ values indicate that the L-HSA binding is an exothermic and entropy decreasing process. And van der Waals force or hydrogen bond interaction plays a major role in this process. The negative signs for $\Delta \mathrm{G}$ reveal that the formation of L-HSA coordination compounds are spontaneous.

\subsection{Conformation investigation}

The synchronous fluorescence spectra are frequently used to characterize the interaction between the molecule probe and proteins since it can provide information about the molecular microenvironment in the vicinity of the chromophore molecules. HSA has intrinsic fluorescence due to there are three intrinsic fluorophores, namely, Trp residues, Tyr residues, and phenylalanine (Phe) residues. Trp and Tyr have the similar excitation spectrum, use the synchronous fluorescence can distinguish them. The synchronous fluorescence spectra reflect the different nature of chromophores: when the $\Delta \lambda$ between excitation wavelength and emission wavelength is set at $60 \mathrm{~nm}$, the synchronous fluorescence spectrum gives the characteristic information of tryptophan residues and when $\Delta \lambda$ value is $15 \mathrm{~nm}$, the synchronous fluorescence spectrum is characteristic of tyrosine residues. Synchronous fluorescence spectra of HSA at various L concentrations when $\Delta \lambda=60 \mathrm{~nm}$ and $\Delta \lambda=15 \mathrm{~nm}$ are shown in Fig. 5. As it is known, nearly all hydrophobic amino acids are embedded in the cylinders forming hydrophobic cavities. When $\Delta \lambda=15 \mathrm{~nm}$, The blue-shift effect suggested that the polarity around the tryptophan residues decreased and the hydrophobicity increased. When $\Delta \lambda=60 \mathrm{~nm}$, the emission peaks show essentially no shift over the investigated concentration range. which indicate that $\mathrm{L}$ have little effect on the microenvironment of tryptophan residues in HSA. 

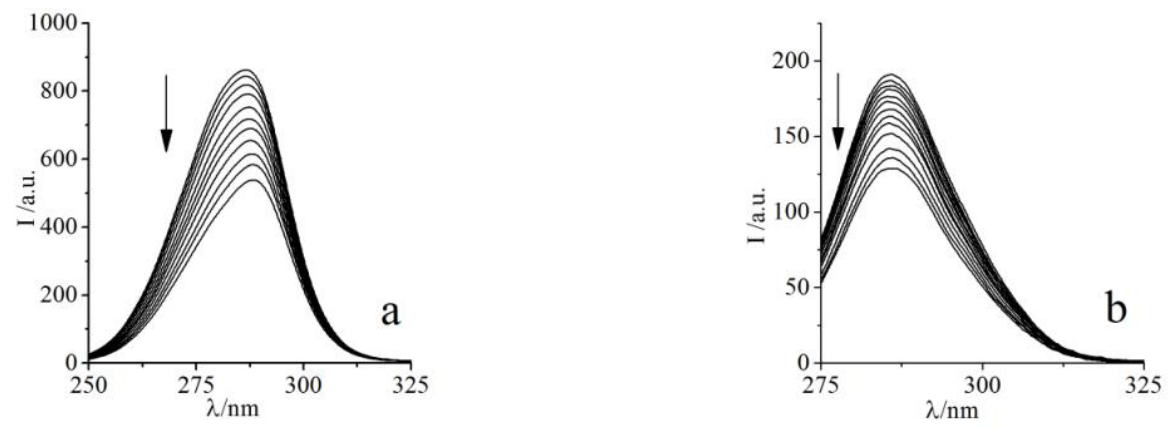

Figure 5. Changes in the synchronous fluorescence spectra of HSA upon increasing concentrations of L (a) $\Delta \lambda=$ $60 \mathrm{~nm}$ and (b) $\Delta \lambda=15 \mathrm{~nm}$.

\section{Conclusions}

The interaction between L and HSA has been investigated under simulated physiological conditions ( $\mathrm{pH} 7.4$, ionic strength $0.1 \mathrm{~mol} / \mathrm{L}$ ) using fluorescence methodology. The quenching rate constant, the binding constants, the number of binding sites, the thermodynamic parameters and the binding forces were obtained. The experimental results indicate that the fluorescence quenching mechanism of HSA by $\mathrm{L}$ was consistent with the static model, and the binding reaction was spontaneous. Van der Waals force or hydrogen bond interaction played a major role in the L-HSA interaction. The results of synchronous fluorescence spectra initiative indicate that the secondary structure of HSA molecules was affected significantly in the presence of L.

\section{References}

1. J. Zhang, H.H. Sun, Y.Z. Zhang, J. Solution Chem, 41 (2012).

2. S.G. Dong, H.M. Cao, L.J. Zhao, Journal of Jilin Medical College, 5 (2014).

3. Y.J. Liu, X.F. Li, H. Fan, L. N. Lu, China Measurement \& Test, 9 (2015).

4. A. Jahanban-Esfahlan, V. Panahi-Azar, Food chemistry, 202 (2016).

5. X. Zheng, M.P. Ye, Y. Li, Journal of China University of Metrology, 1 (2015).

6. W.Y. He, G.Y. Chen, J. Du, Acta Chim. Sinica, 66 (2008).

7. F.L. Cui, J.L. Wang, Y.R. Cui, J.P. Li, Anal. Chim. Acta, 571 (2006) .

8. L. N. Hordge, K. L. McDaniel, D. D. Jones, et al, Talanta, 152 (2016).

9. N. Nanjundan, P. Selvakumar, R. Narayanasamy, et al. J. Photoch. Photobiolo. B, 141 (2014).

10. J. J. Xue, Q. Y. Chen. Spectrochim. Acta A, 120 (2014).

11. F. Samari, M. Shamsipur, B. Hemmateenejad, T. Khayamian, Eur. J. Med. Chem., 54 (2012). 